\title{
Direct experimental test of commutation relation via imaginary weak value
}

\author{
Richard Wagner $\odot,{ }^{1}$ Wenzel Kersten $\odot,{ }^{1}$ Armin Danner $\odot,{ }^{1}$ Hartmut Lemmel $\odot,{ }^{1,2}$ Alok Kumar Pan $\odot,{ }^{3}$ and Stephan Sponar $\odot{ }^{1, *}$ \\ ${ }^{1}$ Atominstitut, TU Wien, Stadionallee 2, 1020 Vienna, Austria \\ ${ }^{2}$ Institut Laue-Langevin, 38000, Grenoble, France \\ ${ }^{3}$ National Institute of Technology Patna, Ashok Rajhpath, Patna 800005, India
}

(Received 26 February 2021; accepted 27 May 2021; published 24 June 2021)

\begin{abstract}
The canonical commutation relation is the hallmark of quantum theory, and Heisenberg's uncertainty relation is a direct consequence of it. Although various formulations of uncertainty relations have been tested in experiments a direct test of the commutation relation has hitherto not been performed. The reason for this is that whereas in quantum mechanics, observables are usually represented by Hermitian operators, the product of two noncommuting observables as occurring in the commutator relation is, in general, non-Hermitian. Here, we present a theoretical scheme for a direct test of the commutation relation and report its experimental realization. More precisely, we provide an experimental verification of the canonical commutation relation between two qubit observables based on a measurement scheme widely known as "weak measurement." In our neutron interferometric setup, we perform the measurement of a single anomalous weak value of a relevant path-qubit observable where the coupled spin-energy degree of freedom serves as an ancilla.
\end{abstract}

DOI: 10.1103/PhysRevResearch.3.023243

\section{INTRODUCTION}

In his epoch-making paper "Quantum-mechanical reinterpretation of kinematic and mechanical relations" in July 1925, Heisenberg [1] put forward his breakthrough idea by introducing an entirely new representation of the position variable in terms of a set of transition amplitudes corresponding to atomic radiation. This led him to propose an unfamiliar rule of multiplication of two amplitudes in order to obtain correct intensities. It was immediately identified as matrix multiplication by Born. In the same year by introducing a mathematically elegant language of matrices, Born and Jordan [2] systematically formalized Heisenberg's "matrix mechanics" and provided the world a one-line epitaph,

$$
\hat{p} \hat{q}-\hat{q} \hat{p}=i \hbar \mathbb{1},
$$

where $\hat{p}$ and $\hat{q}$ are the matrix forms of position and momentum variables in classical mechanics. Equation (1) is now widely known as the canonical commutation relation. The very next year, Schrödinger [3] surprisingly put forward an alternative theory, coined as "wave mechanics" in which the core element is the wave-function $\Psi$. In terms of interpretation and spirit, the wave mechanics greatly differs from the matrix mechanics but produces equivalent quantum statistics.

Two years after his first breakthrough work, in another seminal paper [4], Heisenberg proposed his famous un-

\footnotetext{
*stephan.sponar@tuwien.ac.at

Published by the American Physical Society under the terms of the Creative Commons Attribution 4.0 International license. Further distribution of this work must maintain attribution to the author(s) and the published article's title, journal citation, and DOI.
}

certainty relation $\delta p \delta q \sim h$ which he regarded as a direct mathematical consequence of the canonical commutation rule in Eq. (1). Here $\delta p$ and $\delta q$ are a sort of uncertainties in momentum and position measurements, respectively. Later, building upon Kennard's [5] idea of interpreting the uncertainties as standard deviation, Robertson [6] generalized Heisenberg's preparation uncertainty relation for any two arbitrary observables $\hat{A}$ and $\hat{B}$ so that $\Delta \hat{A} \Delta \hat{B} \geqslant|\langle[\hat{A}, \hat{B}]\rangle| / 2$. In recent times, the distinction between preparation and measurement uncertainty relations has been made, and many interesting new formulations have also been proposed [7-13]. Quite a few of them have experimentally been tested [14-18]. However, no clear consensus among physicists as to the appropriate measure of measurement (in)accuracies has been reached until date $[9,10]$.

Although the uncertainty relations are regarded as direct (or indirect) consequences of relevant commutation relations, only very few attempts have been made to directly test the commutation relations [19-23]. We attribute this to the fact that the joint probability of two noncommuting observables does not exist in quantum theory and the product of such observables is, in general, non-Hermitian. Hence, a direct test of commutation relation is nontrivial in experiment. In this paper, we propose a theoretical scheme in which a single anomalous weak value enables a direct test of the commutation relation between qubit observables. Furthermore, we report an experimental test using neutron interferometry.

The weak value of an observable arises in a novel conditional measurement protocol [24], widely known as "weak measurement." Consider a system prepared in a state $\left|\psi_{\mathrm{i}}\right\rangle$ (commonly known as preselected state) and an observable $\hat{A}$ to be measured on the system. If the measurement interaction between the system and the apparatus is weak, the system state remains grossly undisturbed. If a particular outcome 
$\left|\psi_{\mathrm{f}}\right\rangle$ is selected after such a weak interaction by sequentially performing a strong measurement (postselection), the final pointer state yields the weak value, quantified by the formula [24],

$$
\langle\hat{A}\rangle_{\mathrm{w}}^{\psi_{\mathrm{i}}, \psi_{\mathrm{f}}}=\frac{\left\langle\psi_{\mathrm{f}}|\hat{A}| \psi_{\mathrm{i}}\right\rangle}{\left\langle\psi_{\mathrm{f}} \mid \psi_{\mathrm{i}}\right\rangle} .
$$

Unlike the expectation value $\langle\hat{A}\rangle$, the weak value $\langle\hat{A}\rangle_{\mathrm{w}}^{\psi_{\mathrm{i}}, \psi_{\mathrm{f}}}$ can be beyond the range of eigenvalues and can even be complex. The physical interpretation and implications of complex and large weak values have been widely discussed in the literature (see, for example, Ref. [25]).

A flurry of theoretical [25-32] and experimental works [33-44] on weak measurement have been reported in the past two decades. An anomalous weak value is proven to be beneficial in many practical applications, such as identifying the tiny spin Hall effect [35], detecting very small beam deflections [37] and improving precision in metrology $[43,44]$. Besides, it provided new insights into conceptual foundations of quantum theory [27,29-32], and experiments have been performed to demonstrate for observing quantum trajectories for photons [38] and realizing counterintuitive quantum paradoxes $[36,42,45]$.

In a fundamental experiment by Lundeen et al. [39], a direct measurement of the quantum wave-function $\Psi(x)$ was performed by using weak measurements. Postselecting the system in momentum state $|p\rangle$, the measured weak value of position $\Pi_{x}=|x\rangle\langle x|$ becomes proportional to $\Psi(x)$. Along the same vein, we formulate an interesting scheme in this article so that a single (anomalous) weak value enables a direct experimental test of the canonical commutation relation between two observables in a qubit system. We experimentally performed the measurement of the weak value of a pathqubit observable in our neutron interferometer setup where the spin/energy degree of freedom serves as a pointer. The experimental results are in good agreement with our theoretical prediction and, hence, provide a genuine experimental verification of the canonical commutation relation.

\section{THEORY}

Without loss of generality, consider two noncommuting qubit observables, say $\hat{A}$ and $\hat{B}$ satisfying $\langle\psi|[\hat{A}, \hat{B}]| \psi\rangle \neq 0$ for all $|\psi\rangle$. Since the product $\hat{A} \hat{B}$ may not be Hermitian in general, the traditional von Neumann quantum measurement scheme cannot be carried out, but the weak measurement suffices for our purpose. Non-Hermitian observables may be measured [46] using weak measurements. But our scheme here is direct and fundamentally different as follows. If $\Pi_{A}^{+}=$ $\left|+_{A}\right\rangle\left\langle+_{A}\right|$ and $\Pi_{B}^{+}=\left|+_{B}\right\rangle\left\langle+_{B}\right|$ are positive-eigenvalue projectors of $\hat{A}$ and $\hat{B}$, respectively, then by writing $\hat{A}=2 \Pi_{A}^{+}-\mathbb{1}$ and $\hat{B}=2 \Pi_{B}^{+}-\mathbb{1}$, one has

$$
\begin{aligned}
\langle\psi|[\hat{A}, \hat{B}]| \psi\rangle & =4\left\langle\psi\left|\Pi_{A}^{+} \Pi_{B}^{+}\right| \psi\right\rangle-4\left\langle\psi\left|\Pi_{B}^{+} \Pi_{A}^{+}\right| \psi\right\rangle \\
& =-4\left|\left\langle+_{B} \mid \psi\right\rangle\right|^{2}\left(\left\langle\Pi_{A}^{+}\right\rangle_{\mathrm{w}}^{\psi,+_{B}}-\left\langle\Pi_{A}^{+}\right\rangle_{\mathrm{w}}^{\psi,+_{B} *}\right) \\
& =-8 i\left|\left\langle+_{B} \mid \psi\right\rangle\right|^{2} \operatorname{Im}\left(\left\langle\Pi_{A}^{+}\right\rangle_{\mathrm{w}}^{\psi,+_{B}}\right),
\end{aligned}
$$

where $\left\langle\Pi_{A}^{+}\right\rangle_{\mathrm{w}}^{\psi,+_{B}}$ is the weak value of the projector $\Pi_{A}^{+}$ for a postselected state $\left|{ }_{B}\right\rangle$ and preselected state $|\psi\rangle$ and $\left|\left\langle+_{B} \mid \psi\right\rangle\right|^{2}$ is the probability of successful postselection.
If $\hat{A}=\hat{\sigma}_{z}$ and $\hat{B}=\hat{\sigma}_{x}$, the commutation relation is

$$
\left\langle\psi\left|\left(\hat{\sigma}_{z} \hat{\sigma}_{x}-\hat{\sigma}_{x} \hat{\sigma}_{z}\right)\right| \psi\right\rangle=2 i\left\langle\psi\left|\hat{\sigma}_{y}\right| \psi\right\rangle .
$$

By writing $\hat{\sigma}_{x}=2 \Pi_{x}^{+}-\mathbb{1}, \hat{\sigma}_{y}=2 \Pi_{y}^{+}-\mathbb{1}, \quad$ and $\quad \hat{\sigma}_{z}=$ $2 \Pi_{z}^{+}-\mathbb{1} \quad$ where $\Pi_{x}^{+}=\left|+_{x}\right\rangle\left\langle+_{x}\left|, \Pi_{y}^{+}=\right|+_{y}\right\rangle\left\langle+_{y}\right|, \quad$ and $\Pi_{z}^{+}=\left|+_{z}\right\rangle\left\langle+_{z}\right|$ are the projectors with positive eigenvalue corresponding to the observables $\hat{\sigma}_{x}, \hat{\sigma}_{y}$, and $\hat{\sigma}_{z}$, respectively, from Eq. (4) it follows:

$$
4\left|\left\langle+_{x} \mid \psi\right\rangle\right|^{2} \operatorname{Im}\left(\left\langle\Pi_{z}^{+}\right\rangle_{\mathrm{w}}^{\psi,++_{x}}\right)=-2\left|\left\langle+_{y} \mid \psi\right\rangle\right|^{2}+1,
$$

where $\left\langle\Pi_{z}^{+}\right\rangle_{\mathrm{w}}^{\psi,+_{x}}=\left\langle+_{x}\left|\Pi_{z}^{+}\right| \psi\right\rangle /\left\langle+_{x} \mid \psi\right\rangle$ is the weak value of $\Pi_{z}^{+}$given the pre- and postselected states $|\psi\rangle$ and $\left|{ }_{x}\right\rangle$, respectively. Thus, the imaginary part of a single weak value $\left\langle\Pi_{z}^{+}\right\rangle_{\mathrm{w}}^{\psi,+{ }_{x}}$ is amenable to test the left-hand side of the commutation relation in Eq. (4). For determining the quantity on the right-hand side one requires to separately measure the postselected probability $\left|\left\langle+_{y} \mid \psi\right\rangle\right|^{2}$.

\section{EXPERIMENT}

To determine the weak value, an established neutron interferometric setup [47-50], depicted in Fig. 1(a), is applied. The system is prepared (preselected) and postselected in the path states,

$$
\begin{aligned}
\left|\psi_{\mathrm{i}}(\chi)\right\rangle & =\frac{1}{\sqrt{2}}\left(|I\rangle+e^{-i \chi}|I I\rangle\right) \\
\left|\psi_{\mathrm{f}}\right\rangle & \equiv\left|+_{x}\right\rangle=\frac{1}{\sqrt{2}}(|I\rangle+|I I\rangle),
\end{aligned}
$$

where $|I\rangle$ and $|I I\rangle$ denotes the path eigenstates of the Pauli path observable given by $\sigma_{z}=|I\rangle\langle I|-| I I\rangle\langle I I|$. The imaginary part of the weak value of the path projector $\Pi_{1}$ is expressed as $\operatorname{Im}\left(\left\langle\Pi_{1}\right\rangle_{\mathrm{w}}^{\psi_{\mathrm{i}},+_{x}}\right)=\sin \chi /(2+2 \cos \chi)$ and the probability of successful postselection $\left|\left\langle+_{x} \mid \psi_{\mathrm{i}}(\chi)\right\rangle\right|^{2}=$ $4 \cos ^{2}(\chi / 2)$ with $\left|+_{x}\right\rangle=(|I\rangle+|I I\rangle) / \sqrt{2}$. The quantity on the right-hand side of Eq. (5) is determined by the postselected probability as $-2\left|\left\langle+_{y} \mid \psi_{\mathrm{i}}(\chi)\right\rangle\right|^{2}+1=\sin \chi$ with $\left|+{ }_{y}\right\rangle=(|I\rangle+i|I I\rangle) / \sqrt{2}$.

The experiment was performed at the S18 interferometer beam line at the research reactor of the Institute LaueLangevin in Grenoble, France. A monochromatic neutron beam with a wavelength of $\lambda=0.192 \mathrm{~nm}$ passes through a magnetic prism, which produces two divergent polarized beams with spin states $\left|\uparrow_{z}\right\rangle$ and $\left|\downarrow_{z}\right\rangle$, respectively. For our purpose, only the beam with spin state $\left|\uparrow_{z}\right\rangle$ is adjusted to fulfill the Bragg condition of the down-stream silicon perfect crystal interferometer of triple Laue geometry. The other beam is transmitted in the forward direction and absorbed by a slab of cadmium to reduce the background noise in the detectors. The first crystal plate splits the beam into two paths $|I\rangle$ and $|I I\rangle$. After passing through the first beam splitter, a phase shifter is inserted into the interferometer to tune the relative phase $\chi$ between path $I$ and path $I I$ and prepare the preselected path state $\left|\psi_{\mathrm{i}}(\chi)\right\rangle$. A RF spin manipulator is placed along path $|I\rangle$ to implement the weak measurement by small manipulation of the spin degree of freedom. Both beams are recombined at the third interferometer plate.

Of the two outgoing beams of the interferometer, only the neutrons in the $O$ beam are selected for analysis 
(a)
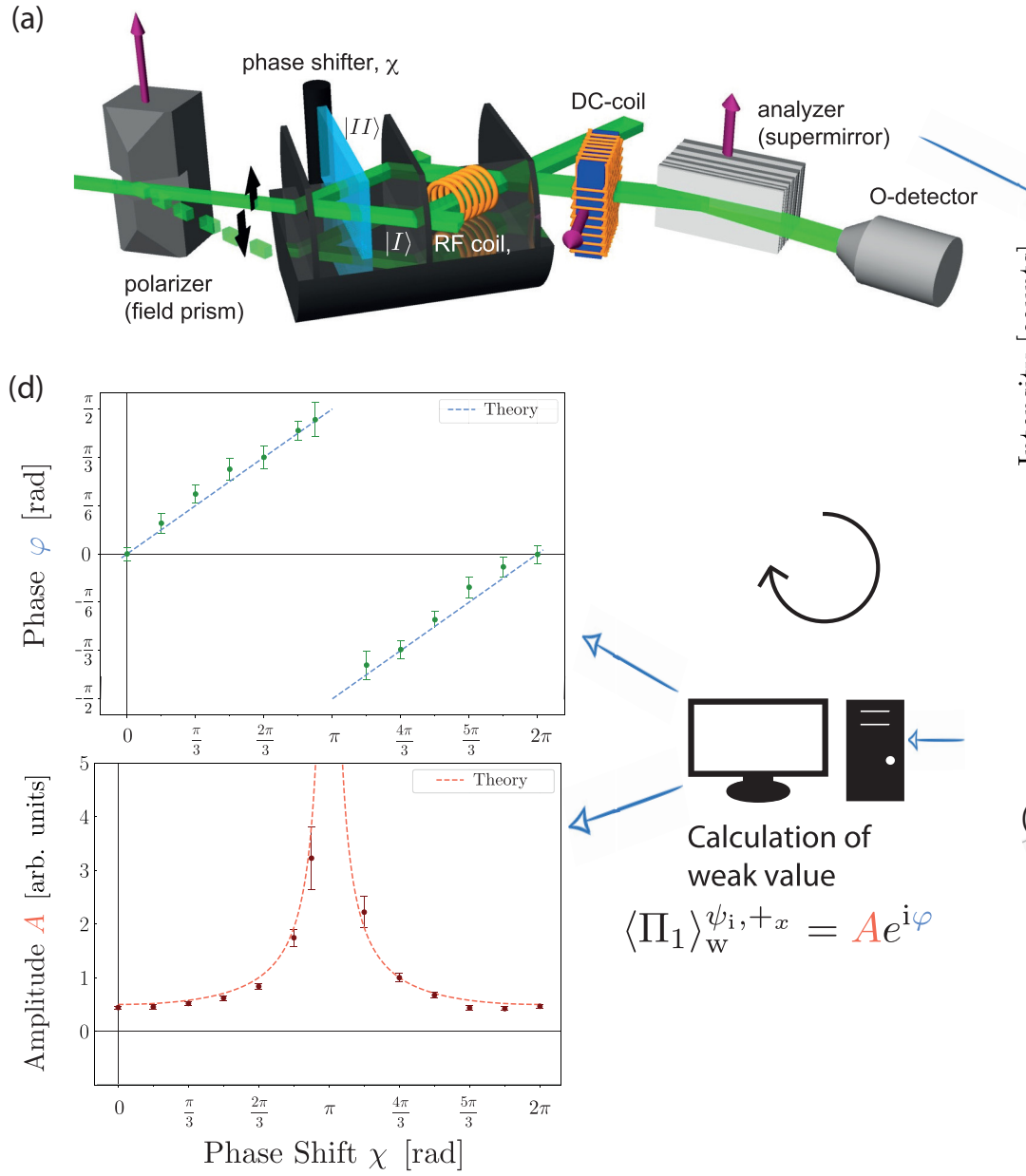

(b)
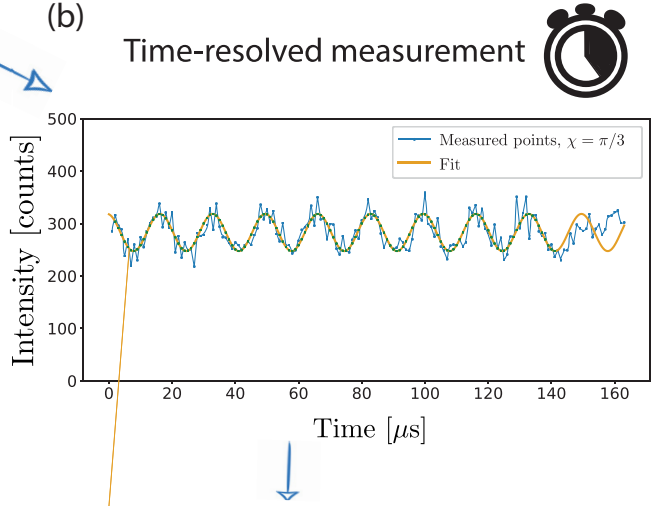

One in set with multiple phase shifter positions

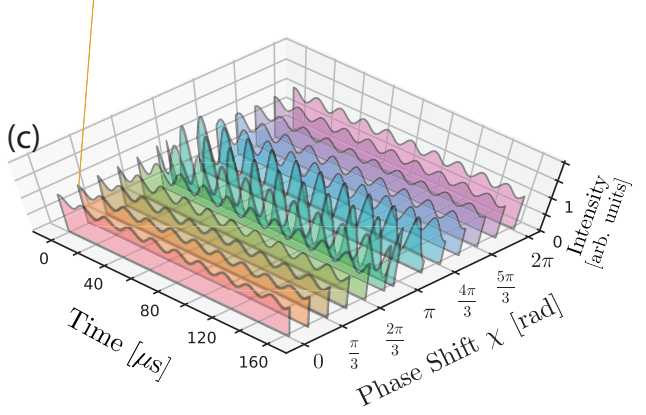

FIG. 1. Illustration of the experimental setup and procedure for the weak measurement of the path state. (a) Neutron interferometric setup: Polarized monochromatic neutrons enter the interferometer and are split into paths $|I\rangle$ and $|I I\rangle$ at the first interferometer plate. Preselection is achieved by adjusting the phase-shifter plate and selecting a corresponding relative phase $\chi$ between the two paths. In path $I$, the neutron beam passes through a resonance frequency (RF) spin flipper, operating at frequency $\omega=60 \mathrm{kHz}$ and is marked by a tiny energy kick of $\hbar \omega$. After postselection of the path state via projection in forward direction at the third interferometer plate, the spin direction is analyzed through the combined action of a direct-current (DC) spin flipper and the supermirror in the $O$ beam. The neutron counts are registered in a ${ }^{3} \mathrm{He}$ countertube. (b) A typical time-resolved intensity signal of the measurement campaign for the phase-shift $\chi=\pi / 3$. (c) Collected time-resolved (normalized) intensity signals for varying phase-shifter position $\chi$. (d) Phase $\varphi$ and amplitude $A$ of the weak value measurement extracted from the time-resolved intensity signals.

corresponding to the postselected path state $\left|\psi_{f}\right\rangle=\left|+_{x}\right\rangle$. The transmitted $O$ beam passes through a DC spin rotator and a polarizing CoTi multilayer array (henceforth referred to as a supermirror) to perform spin analysis in the $O$ beam. The refracted $H$ beam is used as a reference. The intensities in both outgoing beams are measured using ${ }^{3} \mathrm{He}$ counting tubes with a high detection efficiency (over 97\%). The detector for the $O$ beam has a diameter of $6 \mathrm{~mm}$ and is placed directly at the outgoing window of the supermirror. For the used wavelength a time resolution of $3 \mu$ s is achieved.

\section{A. Weak value extraction}

Given the pre- and postselected path states $\left|\psi_{\mathrm{i}}(\chi)\right\rangle$ and $\left|+_{x}\right\rangle$, respectively, we performed the measurement of the weak value $\left\langle\Pi_{1}\right\rangle_{\mathrm{w}}^{\psi_{\mathrm{i}},+_{x}}$ of the path projector $\Pi_{1}=|I\rangle\langle I|$. The weak interaction is implemented by use of the RF spin manipulator in path $|I\rangle$. Its effect can be represented by a unitary evolution as $\mathcal{U}_{\text {int }}=\Pi_{1} \otimes \mathcal{U}_{\mathrm{RF}}(t, \alpha, \omega, \delta)+\Pi_{2}$. Here, $\alpha$ is the spin-rotation angle, $\omega$ is the frequency of the electromagnetic RF field, and $\delta$ is an arbitrary phase of this RF field. The parameter $\alpha$ is directly dependent on the magnetic-field strength, and a sufficiently small value of it warrants the required weak measurement criteria. In this experiment, $\alpha=$ $\pi / 9$ and $\omega=60 \mathrm{kHz}$ were taken. As explained in detail in Appendix A, the weak value $\left\langle\Pi_{1}\right\rangle_{\mathrm{w}}^{\psi_{\mathrm{i}},+_{x}}$ can be extracted from the time-dependent intensity at the $O$ detector that is given by

$$
I(t)=\frac{1}{2}\left|\left\langle+_{x} \mid \psi\right\rangle\right|^{2}\left[1+\alpha \operatorname{Im}\left(e^{i \omega t}\left\langle\Pi_{1}\right\rangle_{\mathrm{w}}^{\psi_{\mathrm{i}},+_{x}}\right)\right]
$$

By switching off the RF field, two postselected probabilities $\left|\left\langle+_{x} \mid \psi\right\rangle\right|^{2}$ and $\left|\left\langle+_{y} \mid \psi_{i}(\chi)\right\rangle\right|^{2}$ are measured. The former resembles an empty interferogram whereas the latter corresponds to an empty but by $\chi_{\text {add }}=-\pi / 2$ shifted interferogram [51]. 


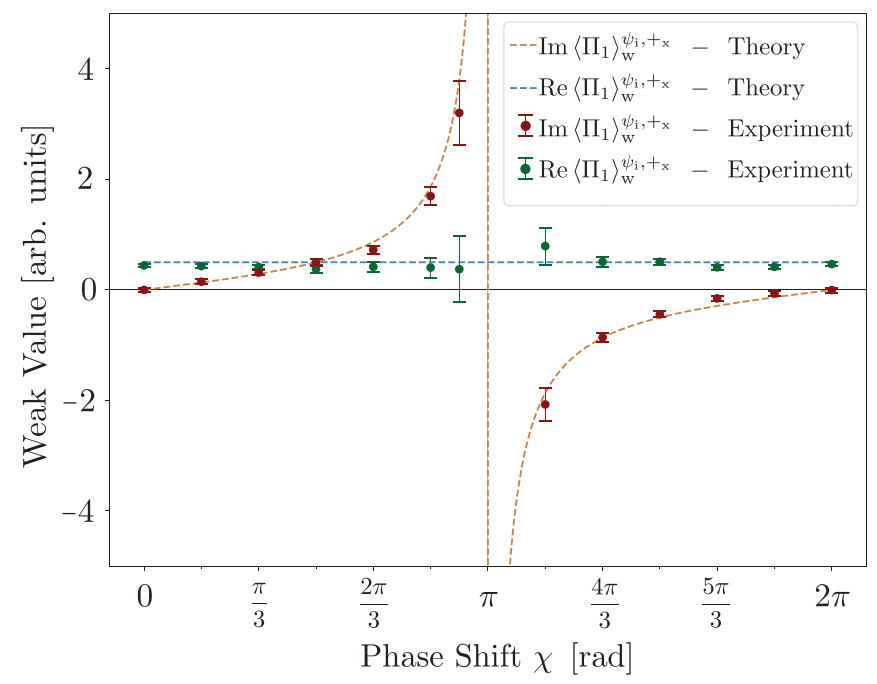

FIG. 2. Experimental results of the real and imaginary parts of weak value $\left\langle\Pi_{1}\right\rangle_{\mathrm{w}}^{\psi_{\mathrm{i}},+_{x}}$ are plotted versus $\chi$. The solid dots denote the least-squares fits to the data, and the error bars represent unit standard deviation.

\section{B. Experimental results}

The weak value is, in general, a complex number and can be expressed in polar form as $\left\langle\Pi_{1}\right\rangle_{\mathrm{w}}^{\psi,+_{x}}=A e^{\mathrm{i} \varphi}$. The amplitude $A$ and phase $\varphi$ are retrieved from a sinusoidal fit to the time-dependent intensity (see Appendix B for details of data analysis). The reference phase $\varphi_{\text {ref }}=0$ is obtained for the particular case when pre- and postselected states coincide, i.e., $\chi=0$. In Fig. 2, we present the detailed analysis of the results of the weak measurement of $\left\langle\Pi_{1}\right\rangle_{\mathrm{w}}^{\psi_{\mathrm{i}},+_{x}}$. For comparison, the experimental values of the real and the imaginary parts of $\left\langle\Pi_{1}\right\rangle_{\mathrm{w}}^{\psi_{\mathrm{i}},+_{x}}$ are separately plotted along with the theoretical predictions $\left\langle\Pi_{1}\right\rangle_{\mathrm{w}}^{\psi_{\mathrm{i}},+_{x}}=\left(1+e^{-i \chi}\right)^{-1}$. As can be seen from the experimental results, the theoretical predictions are reproduced evidently.

Finally, the results of all three measurements, namely, $\left|\left\langle+_{x} \mid \psi\right\rangle\right|^{2},\left|\left\langle+_{y} \mid \psi_{\mathrm{i}}(\chi)\right\rangle\right|^{2}$, and $\operatorname{Im}\left(\left\langle\Pi_{1}\right\rangle_{\mathrm{w}}^{\psi_{\mathrm{i}},+_{x}}\right)$ are combined in Fig. 3 for the direct test of the commutation relation. The leftand right-hand side related measurements of the commutation relation [Eq. (5)] are shown by orange and green data points, respectively. The result is in good agreement with the relevant theoretical prediction (dotted blue), verifying the canonical commutation relation as expressed in Eq. (5).

\section{DISCUSSION}

The results above confirm the canonical commutation relation by weak measurement of the path-qubit observable in a neutron interferometer. Accordingly, from the quantum foundational perspective, our experiment, thus, provides a direct test of one of the fundamental tenets of quantum theory. This test is as fundamental as the direct measurement of a quantum wave function by Lundeen et al. [39]. Heisenberg's uncertainty relation may be regarded as a direct consequence of the canonical commutation relation and several formulations of it have been tested experimentally [14,15,52-54].

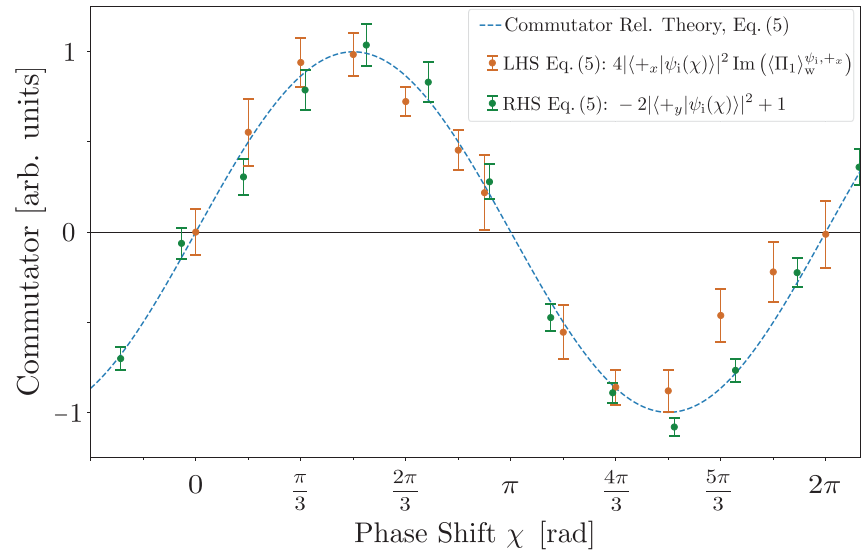

FIG. 3. Experimental results of left-hand side (orange) and righthand side (green) of the commutation relation in Eq. (5) are plotted as a function of phase-shift $\chi$. The results are in excellent agreement with the relevant theoretical prediction (dotted blue). The error bars represent one standard deviation.

At this point we want to emphasize that our presented scheme is fundamentally different compared to prior experiments studying the commutation relation [19-21]. In these experiments the noncommutativity of Pauli spin matrices in terms of different sequences of rotations (on the same initial state) was utilized. However, a direct test of the canonical commutation relation should unambiguously determine the expectation value of the non-Hermitian product of two noncommuting observables as it occurs in the commutation relation. As presented here, the direct test of the commutation relation boils down to the experimental determination of the imaginary part of a single weak value of a suitable path observable. The weak value has provided a multitude of practical and conceptual implications [25-44,46,55]. The imaginary part of the weak value is less intuitive than its real counterpart. The latter can be operationally interpreted as an idealized conditioned average value of the concerned observable in the zero measurement disturbance limit. On the other hand, the imaginary part is associated with backaction on the system due to the measurement itself [55]. This explanation conceptually fits well with our test of the commutation relation through the imaginary part of the weak value. Finally, it would be interesting to test the traditional position-momentum uncertainty relation following the approach developed here. The experimental method presented here could be expanded to more path markers in the interferometer, and several path weak values could be measured simultaneously. Applying this procedure to a four-plate interferometer would even offer more than two path markers [50].

\section{ACKNOWLEDGMENTS}

This work was financed by the Austrian Science Fund (FWF), Project No. P30677. A.K.P. acknowledges support from Project No. DST/ICPS/QuST/Theme-1/2019/4. 


\section{APPENDIX A: DETAILED ANALYSIS OF THE INTENSITY FOR THE WEAK VALUE EXTRACTION}

Given the preselected path state denoted as $\left|\psi_{\mathrm{i}}(\chi)\right\rangle=$ $\frac{1}{\sqrt{2}}\left(|I\rangle+e^{-\mathrm{i} \chi}|I I\rangle\right)$ and postselected path state $\left|\psi_{\mathrm{f}}\right\rangle=$ $\frac{1}{\sqrt{2}}(|I\rangle+|I I\rangle)$ to determine the weak value [26,49] of path observable $\Pi_{1}$, an interaction of the RF spin manipulator in path $|I\rangle$ is employed which can be represented by the unitary dynamics, denoted as $\mathcal{U}_{\text {int }}=\Pi_{1} \otimes \mathcal{U}_{\mathrm{RF}}(t, \alpha, \omega, \delta)+\Pi_{2}$. The spin manipulation [50] leads to an energy shift by the amount $\Delta E= \pm \hbar \omega$ in the spin-flipped parts and can be written as a unitary operator,

$$
\mathcal{U}_{\mathrm{RF}}(t, \alpha, \omega, \delta)=\left(\begin{array}{cc}
\cos \left(\frac{\alpha}{2}\right) & i \sin \left(\frac{\alpha}{2}\right) e^{+i(\omega t+\delta)} \\
i \sin \left(\frac{\alpha}{2}\right) e^{-i(\omega t+\delta)} & \cos \left(\frac{\alpha}{2}\right)
\end{array}\right),
$$

where $\alpha, \omega$, and $\delta$ are the spin-rotation angle, the frequency of the electromagnetic RF field and an arbitrary phase of this RF field. The energy-shifted contributions appear due to the off-diagonal terms. The parameter $\alpha$ is related to magneticfield strength and for a small value of $\alpha$ (A1) becomes

$$
\lim _{\alpha \rightarrow 0} \mathcal{U}_{\mathrm{RF}}=\mathbb{1}-i \frac{\alpha}{2}\left(\begin{array}{cc}
0 & e^{+i(\omega t+\delta)} \\
e^{-i(\omega t+\delta)} & 0
\end{array}\right) .
$$

The total path-spin state of the in $\uparrow_{z}$-direction polarized neutrons after the first plate of the interferometer is

$$
|\Psi\rangle=\left|\psi_{\mathrm{i}}(\chi)\right\rangle \otimes\left|\uparrow_{z}\right\rangle .
$$

Spin-related states and projectors are indicated by the $\uparrow$ symbol. After the interaction with the spin modulator in path $I$, the state evolves to $\left|\Psi^{\prime}\right\rangle=\mathcal{U}_{\text {int }}|\Psi\rangle$ and is subsequently projected onto the postselected path state $\left|\psi_{\mathrm{f}}\right\rangle$ at the third crystal plate,

$$
\left|\Psi^{\prime}\right\rangle \rightarrow\left|\Psi^{\prime \prime}\right\rangle=\Pi_{\mathrm{f}} \mathcal{U}_{\text {int }}|\Psi\rangle,
$$

where $\Pi_{\mathrm{f}}=\left|\psi_{\mathrm{f}}\right\rangle\left\langle\psi_{\mathrm{f}}\right|$. Now, for our purpose, the spin analysis is performed for $\left|\uparrow_{x}\right\rangle$ which is implemented by the action of the DC coil and the supermirror. The joint (un-normalized) path-spin state is projected onto $P_{\uparrow_{x}}=\left|\uparrow_{x}\right\rangle\left\langle\uparrow_{x}\right|$,

$$
\left|\Psi^{\prime \prime \prime}\right\rangle=P_{\uparrow_{x}} \Pi_{\mathrm{f}} \mathcal{U}_{\text {int }}|\Psi\rangle .
$$

Using (A2) we get the approximation in the limit for small $\alpha$ [neglecting contributions on the order of $O\left(\alpha^{2}\right)$ or higher],

$$
\left|\Psi^{\prime \prime \prime}\right\rangle \approx \frac{1}{\sqrt{2}}\left|\psi_{\mathrm{f}}\right\rangle \otimes\left|\uparrow_{x}\right\rangle\left\langle\psi_{\mathrm{f}}\left|\left[\mathbb{1}-i \frac{\alpha}{2}\left(\Pi_{1} e^{i(\omega t+\delta)}\right)\right]\right| \psi_{\mathrm{i}}(\chi)\right\rangle .
$$

Further rearrangement provides

$$
\begin{aligned}
\left|\Psi^{\prime \prime \prime}\right\rangle= & \frac{1}{\sqrt{2}}\left(\left|\psi_{\mathrm{f}}\right\rangle \otimes\left|\uparrow_{x}\right\rangle\right)\left\langle\psi_{\mathrm{f}} \mid \psi_{\mathrm{i}}(\chi)\right\rangle \\
& \times\left[1-i \frac{\alpha}{2}\left(\left\langle\Pi_{1}\right\rangle_{w}^{\psi_{\mathrm{i}}, \psi_{\mathrm{f}}} e^{\mathrm{i}(\omega t+\delta)}\right)\right] .
\end{aligned}
$$

The time-dependent intensity $I_{\text {ideal }}(t)=\left|\Psi^{\prime \prime \prime}\right|^{2}$ in the ideal scenario can then be written as

$$
I_{\text {ideal }}(t)=\frac{1}{2}\left|\left\langle\psi_{\mathrm{f}} \mid \psi_{\mathrm{i}}(\chi)\right\rangle\right|^{2}\left[1+\alpha \operatorname{Im}\left(\left\langle\Pi_{1}\right\rangle_{w}^{\psi_{\mathrm{i}}, \psi_{\mathrm{f}}} e^{i(\omega t+\delta)}\right)\right],
$$

which is dependent on $\chi$. With $\left|\psi_{\mathrm{f}}\right\rangle \equiv\left|+_{x}\right\rangle$ the weak value of the projector onto path $I$, given by $\left\langle\Pi_{1}\right\rangle_{\mathrm{w}}^{\psi_{\mathrm{i}},+_{x}}=(1+$ $\left.e^{-\mathrm{i} \chi}\right)^{-1}$, can be extracted from the intensity analysis. By switching off the RF spin modulator $(\alpha=0)$, the signal for postselected probabilities $\left|\left\langle+_{x} \mid \psi_{\mathrm{i}}(\chi)\right\rangle\right|^{2}=(1+\cos \chi) / 2$ and $\left|\left\langle+_{y} \mid \psi_{i}(\chi)\right\rangle\right|^{2}=(1-\sin \chi) / 2$ in Eq. (5) can be measured. For the latter, in practice this is performed by applying an additional phase shift of $\chi_{\text {add }}=-\pi / 2$ to the readings from the measurement of the empty interferogram [51].

\section{APPENDIX B: DATA ANALYSIS}

In experiments, there inevitably occurs a loss of coherence in the signal. Therefore, the intensity has to be corrected by carefully taking into account the incoherent contributions. The amount of this incoherence can be accounted for by the contrast or fringe visibility parameter $\eta \in[0,1]$. The intensity then reads as

$$
I_{\text {real }}=\eta I_{\text {ideal }}+(1-\eta) I_{\text {inc }},
$$

where $I_{\text {inc }}$ stands for its incoherent part. By considering an empty interferometer, we quantify $I_{\text {ideal }}^{\text {empty }}$ as

$$
\begin{aligned}
I_{\text {ideal }}^{\text {empty }} & =\left|\left\langle\psi_{\mathrm{f}} \mid \psi_{\mathrm{i}}\right\rangle\right|^{2}=|\underbrace{\langle I \mid I\rangle}_{\Psi_{1}}+\underbrace{e^{\mathrm{i} \chi}\langle I I \mid I I\rangle}_{\Psi_{2}}|^{2} \\
& =\left|\Psi_{1}\right|^{2}+\left|\Psi_{2}\right|^{2}+2 \operatorname{Re}\left(\Psi_{1}^{*} \Psi_{2}\right) .
\end{aligned}
$$

In an ideal scenario we expect full coherence of the interference term $2 \operatorname{Re}\left(\Psi_{1}^{*} \Psi_{2}\right)$, i.e., $\eta=1$. But, in real experiment, partial coherence can be obtained so that in our experiment $I_{\text {real }}^{\text {empty }}$ is quantified as

$$
\begin{aligned}
I_{\text {real }}^{\text {empty }} & =\left|\Psi_{1}\right|^{2}+\left|\Psi_{2}\right|^{2}+2 \eta \operatorname{Re}\left(\Psi_{1}^{*} \Psi_{2}\right) \\
& =\eta\left|\Psi_{1}+\Psi_{2}\right|^{2}+(1-\eta)\left[\left|\Psi_{1}\right|^{2}+\left|\Psi_{2}\right|^{2}\right] .
\end{aligned}
$$

Identifying the incoherent part as $I_{\text {inc }}=\left|\Psi_{1}\right|^{2}+\left|\Psi_{2}\right|^{2}$, it is straightforward to see that Eq. (B3) is equivalent to Eq. (B1). The time-dependent intensity in our experiment can then be written as

$$
\begin{aligned}
I_{\text {real }}(t)= & \frac{\eta}{2}\left|\left\langle\psi_{\mathrm{f}} \mid \psi_{\mathrm{i}}(\chi)\right\rangle\right|^{2}\left[1+\alpha \operatorname{Im}\left(\left\langle\Pi_{1}\right\rangle_{\mathrm{w}} e^{i(\omega t+\delta)}\right)\right] \\
& +(1-\eta)(\underbrace{\frac{1}{8}[1-\alpha \sin (\omega t+\delta)]}_{\left|\Psi_{1}\right|^{2}:=I_{1}(t)}+\underbrace{\frac{1}{8}}_{\left|\Psi_{2}\right|^{2}:=I_{2}(t)}),
\end{aligned}
$$

$\left|\Psi_{1}\right|^{2}$ and $\left|\Psi_{2}\right|^{2}$ are intensities for the isolated paths $I$ and $I I$, respectively, which are measured separately to apply the correction. An example of such a signal is shown in Fig. 1(b). The visibility $\eta$ is extracted from an interferogram of an empty interferometer by fitting according to Eq. (B3). In our measurements the average value of $\eta$ is 0.79 .

Now, the ideal intensity is the one that is the measured intensity minus contributions from independent (decoherent) intensities,

$$
I_{\text {ideal }}(t)=\frac{1}{\eta}\left\{I_{\text {real }}(t)-(1-\eta)\left[I_{1}(t)+I_{2}(t)\right]\right\} .
$$

Time-resolved signals have been recorded for several phaseshifter settings in the range of $0 \leqslant \chi \leqslant 2 \pi$. These have been corrected according to Eq. (B5), fitted and normalized. The 
time-resolved signals are shown in Fig. 1(b) for $\chi=\pi / 3$ and in Fig. 1(c) for all values of $\chi$.

The weak value for the path projector is a complex number and can generally be written in the polar form $\left\langle\Pi_{1}\right\rangle_{\mathrm{w}}^{\psi_{\mathrm{i}},+_{x}}=$ $A e^{i \varphi}$. So it follows from Eq. (A8) that its amplitude and phase can be obtained from sine fits to the time-dependent intensities,

$$
I_{\text {ideal }}(t)=\frac{1}{2}\left|\left\langle\psi_{\mathrm{f}} \mid \psi_{\mathrm{i}}(\chi)\right\rangle\right|^{2}[1+\alpha A \sin (\varphi-\omega t-\delta)] .
$$

Here $\delta$ is an arbitrary additional constant phase. The reduction of the fringe visibility applies also to Eq. (B6). The retrieved values for the amplitudes $A$ have, therefore, to be corrected with $\eta$, according to $A_{\mathrm{w}}=A / \eta$ to obtain the amplitudes of the path projector weak values. At the phase-shifter setting $\chi=$ 0 , the weak value $\left\langle\Pi_{1}\right\rangle_{\mathrm{w}}^{\psi_{\mathrm{i}},{ }_{x}}=\left(1+e^{-i \chi}\right)^{-1}$ becomes real and exactly $1 / 2$. So the phase angle $\varphi$ is equal to zero. This serves as a reference in the measurement to retrieve the phase angle $\varphi$ for the other $\chi$ settings. A plot that collects the retrieved amplitudes $A_{\mathrm{w}}$ and phases $\varphi$ for the weak value of the path projector $\left\langle\Pi_{1}\right\rangle_{\mathrm{w}}^{\psi_{\mathrm{i}},+_{x}}$ is shown in Fig. 1(d).

The errors in all plots are from propagation of the standard deviations of the fit errors for the time-dependent intensity, reference interferogram signals, and the systematic effects due to uncertainties in the adjustment of the rotation angle $\alpha$.
[1] W. Heisenberg, Über quantentheoretische Umdeutung kinematischer und mechanischer Beziehungen, english translation: Quantum mechanical reinterpretation of kinematic and mechanical relations, Z. Phys. 33, 879 (1925).

[2] M. Born and P. Jordan, Zur Quantenmechanik, Z. Phys. 34, 858 (1925).

[3] E. Schrödinger, An undulatory theory of the mechanics of atoms and molecules, Phys. Rev. 28, 1049 (1926).

[4] W. Heisenberg, Über den anschaulichen Inhalt der quantentheoretischen Kinematik und Mechanik, english translation: The physical content of quantum kinematics and mechanics, $\mathrm{Z}$. Phys. 43, 172 (1927).

[5] E. H. Kennard, Zur Quantenmechanik einfacher Bewegungstypen, Z. Phys. 44, 326 (1927).

[6] H. P. Robertson, The Uncertainty principle, Phys. Rev. 34, 163 (1929).

[7] E. Arthurs and J. L. Kelly, On the simultaneous measurement of a pair of conjugate observables, Bell Syst. Tech. J. 44, 725 (1965).

[8] P. Busch, Indeterminacy relations and simultaneous measurements in quantum theory, Int. J. Theor. Phys. 24, 63 (1985).

[9] M. Ozawa, Physical content of Heisenberg's uncertainty relation: limitation and reformulation, Phys. Lett. A 318, 21 (2003).

[10] P. Busch, P. Lahti, and R. F. Werner, Proof of Heisenberg's Error-Disturbance Relation, Phys. Rev. Lett. 111, 160405 (2013).

[11] P. Busch, P. Lahti, and R. F. Werner, Colloquium: Quantum root-mean-square error and measurement uncertainty relations, Rev. Mod. Phys. 86, 1261 (2014).

[12] F. Buscemi, M. J. W. Hall, M. Ozawa, and M. M. Wilde, Noise and Disturbance in Quantum Measurements: An InformationTheoretic Approach, Phys. Rev. Lett. 112, 050401 (2014).

[13] C. Branciard, Error-tradeoff and error-disturbance relations for incompatible quantum measurements, Proc. Natl. Acad. Sci. USA 110, 6742 (2013).

[14] J. Erhart, S. Sponar, G. Sulyok, G. Badurek, M. Ozawa, and Y. Hasegawa, Experimental demonstration of a universally valid error-disturbance uncertainty relation in spin-measurements, Nat. Phys. 8, 185 (2012).

[15] L. A. Rozema, A. Darabi, D. H. Mahler, A. Hayat, Y. Soudagar, and A. M. Steinberg, Violation of Heisenberg's MeasurementDisturbance Relationship by Weak Measurements, Phys. Rev. Lett. 109, 100404 (2012).
[16] S. Sponar, G. Sulyok, J. Erhart, and Y. Hasegawa, Errordisturbance uncertainty relations in neutron-spin measurements, Adv. High Energy Phys. 2014, 735398 (2015).

[17] G. Sulyok, S. Sponar, B. Demirel, F. Buscemi, M. J. W. Hall, M Ozawa, and Y. Hasegawa, Experimental Test of Entropic NoiseDisturbance Uncertainty Relations for Spin-1/2 Measurements, Phys. Rev. Lett. 115, 030401 (2015).

[18] B. Demirel, S. Sponar, A. A. Abbott, C. Branciard, and Y. Hasegawa, Experimental test of an entropic measurement uncertainty relation for arbitrary qubit observables, New J. Phys. 21, 013038 (2019).

[19] Y. Hasegawa, S. Menhart, R. Meixner, and G. Badurek, Noncommutation of Pauli spin operators in neutron polarimetry, Phys. Lett. A 234, 322 (1997).

[20] A. G. Wagh, V. C. Rakhecha, J. Summhammer, G. Badurek, H. Weinfurter, B. E. Allman, H. Kaiser, K. Hamacher, D. L. Jacobson, and S. A. Werner, Experimental Separation of Geometric and Dynamical Phases using Neutron Interferometry, Phys. Rev. Lett. 78, 755 (1997).

[21] Y.-S. Kim, H.-T. Lim, Y.-S. Ra, and Y.-H. Kim, Experimental verification of the commutation relation for pauli spin operators using single-photon quantum interference, Phys. Lett. A 374, 4393 (2010).

[22] A. Zavatta, V. Parigi, M. S. Kim, H. Jeong, and M. Bellini, Experimental Demonstration of the Bosonic Commutation Relation via Superpositions of Quantum Operations on Thermal Light Fields, Phys. Rev. Lett. 103, 140406 (2009).

[23] X.-C. Yao, J. Fiurášek, H. Lu, W.-B. Gao, Y.-A. Chen, Z.-B. Chen, and J.-W. Pan, Experimental Realization of Programmable Quantum Gate Array for Directly Probing Commutation Relations of Pauli Operators, Phys. Rev. Lett. 105, 120402 (2010).

[24] Y. Aharonov, D. Z. Albert, and L. Vaidman, How the Result of a Measurement of a Component of the Spin of a Spin-1/2 Particle can Turn out to be 100, Phys. Rev. Lett. 60, 1351 (1988).

[25] J. Dressel, M. Malik, F. M. Miatto, A. N. Jordan, and R. W. Boyd, Colloquium: Understanding quantum weak values: Basics and applications, Rev. Mod. Phys. 86, 307 (2014).

[26] I. M. Duck, P. M. Stevenson, and E. C. G. Sudarshan, The sense in which a "weak measurement" of a spin-1/2 particle's spin component yields a value 100, Phys. Rev. D 40, 2112 (1989).

[27] Y. Aharonov and L. Vaidman, Complete description of a quantum system at a given time, J. Phys. A 24, 2315 (1991). 
[28] K. J. Resch and A. M. Steinberg, Extracting Joint Weak Values with Local, Single-Particle Measurements, Phys. Rev. Lett. 92, 130402 (2004).

[29] M. F. Pusey, Anomalous Weak Values are Proofs of Contextuality, Phys. Rev. Lett. 113, 200401 (2014).

[30] L. Vaidman, Past of a quantum particle, Phys. Rev. A 87, 052104 (2013).

[31] Y. Aharonov, S. Popescu, D. Rohrlich, and P. Skrzypczyk, Quantum Cheshire Cats, New J. Phys. 15, 113015 (2013).

[32] Y. Aharonov, F. Colombo, S. Popescu, I. Sabadini, D. C. Struppa, and J. Tollaksen, Quantum violation of the pigeonhole principle and the nature of quantum correlations, Proc. Natl. Acad. Sci. USA 113, 532 (2016).

[33] N. W. M. Ritchie, J. G. Story, and R. G. Hulet, Realization of a Measurement of a "weak value", Phys. Rev. Lett. 66, 1107 (1991).

[34] G. J. Pryde, J. L. O'Brien, A. G. White, T. C. Ralph, and H. M. Wiseman, Measurement of Quantum Weak Values of Photon Polarization, Phys. Rev. Lett. 94, 220405 (2005).

[35] O. Hosten and P. Kwiat, Observation of the spin Hall effect of light via weak measurements, Science 319, 787 (2008).

[36] J. S. Lundeen and A. M. Steinberg, Experimental Joint Weak Measurement on a Photon Pair as a Probe of Hardy's Paradox, Phys. Rev. Lett. 102, 020404 (2009).

[37] D. J. Starling, P. B. Dixon, A. N. Jordan, and J. C. Howell, Optimizing the signal-to-noise ratio of a beam-deflection measurement with interferometric weak values, Phys. Rev. A 80, 041803(R) (2009).

[38] S. Kocsis, B. Braverman, S. Ravets, M. J. Stevens, R. P. Mirin, L. K. Shalm, and A. M. Steinberg, Observing the average trajectories of single photons in a two-slit interferometer, Science 332, 1170 (2011).

[39] J. S. Lundeen, B. Sutherland, A. Patel, C. Stewart, and C. Bamber, Direct measurement of the quantum wavefunction, Nature (London) 474, 188 (2011).

[40] A. Danan, D. Farfurnik, S. Bar-Ad, and L. Vaidman, Asking Photons where they have been, Phys. Rev. Lett. 111, 240402 (2013).

[41] F. Piacentini, A. Avella, M. P. Levi, M. Gramegna, G. Brida, I. P. Degiovanni, E. Cohen, R. Lussana, F. Villa, A. Tosi, F. Zappa, and M. Genovese, Measuring Incompatible Observables by Exploiting Sequential Weak Values, Phys. Rev. Lett. 117, 170402 (2016).

[42] T. Denkmayr, H. Geppert, S. Sponar, H. Lemmel, A. Matzkin, J. Tollaksen, and Y. Hasegawa, Experimental observation of a quantum Cheshire Cat in matter-wave interferometry, Nat. Commun. 5, 4492 (2014).

[43] L. Xu, Z. Liu, A. Datta, G. C. Knee, J. S. Lundeen, Y.-q. Lu, and L. Zhang, Approaching Quantum-Limited Metrology with Imperfect Detectors by using Weak-Value Amplification, Phys. Rev. Lett. 125, 080501 (2020).

[44] D. R. M. Arvidsson-Shukur, N. Yunger Halpern, H. V. Lepage, A. A. Lasek, C. H. W. Barnes, and S. Lloyd, Quantum advantage in postselected metrology, Nat. Commun. 11, 3775 (2020).

[45] X.-J. Chen, W.-N. Zhang, B. Chen, W.-D. Xi, Y. Lu, J.Y. Huang, Y.-Y. Wang, J. Long, S.-F. Wu, Y.-X. Zhang, S. Wang, S.-X. Li, T. Yin, M. Lu, X.-D. Xi, J.-M. Li, K.-K. Wang, Z. Chen, and S.-J. Chen, Homoharringtonine deregulates myc transcriptional expression by directly binding NF- $\kappa \mathrm{B}$ repressing factor, Proc. Natl. Acad. Sci. USA 116, 2220 (2019).

[46] G. Nirala, S. N. Sahoo, A. K. Pati, and U. Sinha, Measuring average of non-hermitian operator with weak value in a MachZehnder interferometer, Phys. Rev. A 99, 022111 (2019).

[47] H. Rauch, W. Treimer, and U. Bonse, Test of a single crystal neutron interferometer, Phys. Lett. A 47, 369 (1974).

[48] S. Sponar, T. Denkmayr, H. Geppert, H. Lemmel, A. Matzkin, J. Tollaksen, and Y. Hasegawa, Weak values obtained in matterwave interferometry, Phys. Rev. A 92, 062121 (2015).

[49] T. Denkmayr, H. Geppert, H. Lemmel, M. Waegell, J. Dressel, Y. Hasegawa, and S. Sponar, Experimental Demonstration of Direct Path State Characterization by Strongly Measuring Weak Values in a Matter-Wave Interferometer, Phys. Rev. Lett. 118 010402 (2017).

[50] H. Geppert-Kleinrath, T. Denkmayr, S. Sponar, H. Lemmel, T. Jenke, and Y. Hasegawa, Multifold paths of neutrons in the three-beam interferometer detected by a tiny energy kick, Phys. Rev. A 97, 052111 (2018).

[51] H. Rauch and S. A. Werner, Neutron Interferometry (Clarendon, Oxford, 2000).

[52] C. G. Shull, Single-slit diffraction of neutrons, Phys. Rev. 179 , 752 (1969).

[53] H. P. Yuen, Two-photon coherent states of the radiation field, Phys. Rev. A 13, 2226 (1976).

[54] G. Breitenbach, S. Schiller, and J. Mlynek, Measurement of the quantum states of squeezed light, Nature (London) 387, 471 (1997).

[55] J. Dressel and A. N. Jordan, Significance of the imaginary part of the weak value, Phys. Rev. A 85, 012107 (2012). 